\title{
How to treat a Rayleigh-like isotope fractionation process when the equilibrium is not reached?
}

\author{
ZIXUAN GUAN $^{1,2}$, PING GUAN ${ }^{1}$, YUN LIU ${ }^{2}$
}

${ }^{1}$ Peking University, Key Lab Orogen Belts \& Crustal Evolut, Minist Educ, Sch Earth \& Space Sci, Beijing, China(Email:pguanl@pku.edu.cn)

${ }^{2}$ State Key Labrotary of Ore Deposit Geochemistry, Institute of Geochemisry, Chinese Academy of Sciences, Guiyang, China (E-mail: guanzixuan2016@pku.edu.cn, liuyun@vip.gyig.ac.cn)

Rayleigh distillation fractionation model is one of the principal tools used for interpreting isotopic fractionations in various geologic processes. However, it requires materials, which are separated from the first reservoir to the second one bit by bit, are kept equilibrium with those remained in the first reservoir. This requirement let Rayleigh fractionation model fail for numerous situations. How to explain isotopic signals preserved in the samples experienced such nonequilibrium separation processes becomes a challenge for isotope geochemistry.

Here we provide a diffusion-coupled Rayleigh-like isotope fractionation model for this issue. Our model treats the isotope fractionation when the diffusion process and the Rayleigh-like separation process act simultaneously. It is like a transportation-fractionation model used for hydrology. We develop our own code for this model, which is specially designed to treat the isotope fractionations. According to the calculations in this study, the reaction factor estimated by the standard Rayleigh fractionation model only indicates the fractionations at the final stages of separation when the scale, or thickness, reduced to the magnitude of the quotient of diffusion rate and separation rate. The Rayleigh fractionation model may fail in the earlier stages, and the overwhelming effect can eventually be the diffusion effect when the system is with a relatively large scale, a fast separation rate, and a low diffusion rate. The influence of internal unevenness caused by diffusion on the Rayleigh-like separation fractionation must be considered.

Our model and its results can be applied to studies of many important issues including partial melting, evaporation, precipitation and core segregation processes. Further information can be extracted from isotopic signals of the samples experienced "disequilibrium" processes based on our model. 